Coulomb asymmetry and sub-cycle electron dynamics in multiphoton multiple ionization of $\mathrm{H}_{2}$

This article has been downloaded from IOPscience. Please scroll down to see the full text article.

2012 J. Phys. B: At. Mol. Opt. Phys. 45194011

(http://iopscience.iop.org/0953-4075/45/19/194011)

View the table of contents for this issue, or go to the journal homepage for more

Download details:

IP Address: 132.246.118.152

The article was downloaded on 01/10/2012 at 18:11

Please note that terms and conditions apply. 


\title{
Coulomb asymmetry and sub-cycle electron dynamics in multiphoton multiple ionization of $\mathbf{H}_{2}$
}

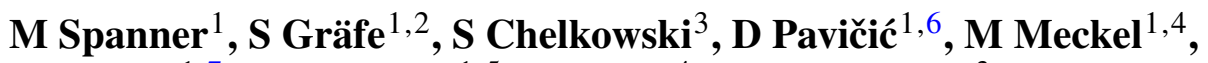 \\ D Zeidler $^{1,7}$, A B Bardon ${ }^{1,5}$, B Ulrich ${ }^{4}$, A D Bandrauk ${ }^{3}$, D M Villeneuve ${ }^{1}$, \\ R Dörner ${ }^{4}$, P B Corkum ${ }^{1}$ and A Staudte ${ }^{1}$ \\ ${ }^{1}$ Joint Laboratory for Attosecond Science of the National Research Council, University of Ottawa, \\ 100 Sussex Drive, Ottawa, Ontario, K1A 0R6 Canada \\ ${ }^{2}$ Institute for Theoretical Physics, Vienna University of Technology, Wiedner Hauptstr. 8-10, \\ A-1040 Vienna, Austria \\ ${ }^{3}$ Département de Chimie, Université de Sherbrooke, Sherbrooke, Québec, J1K 2R1 Canada \\ ${ }^{4}$ Institut für Kernphysik, Goethe Universität, Max-von-Laue Str. 1, D-60438 Frankfurt, Germany \\ ${ }^{5}$ Department of Physics, University of Toronto, 60 Saint George St, Toronto, Ontario, M5S 1A7 Canada \\ E-mail: michael.spanner@nrc.ca and andre.staudte@nrc.ca
}

Received 16 May 2012, in final form 21 June 2012

Published 24 September 2012

Online at stacks.iop.org/JPhysB/45/194011

\begin{abstract}
We present a systematic study of the molecular-frame photo-electron angular distributions produced by multiphoton double ionization of $\mathrm{H}_{2}$ using circularly polarized $800 \mathrm{~nm}$, femtosecond laser pulses. We compare experimental results to numerical results obtained from a reduced-dimensionality time-dependent Schrödinger equation (TDSE) model. In addition, we implement a TDSE-like version of the strong-field approximation to isolate the effect of the parent ion's Coulomb potential on the continuum electron in our simulations. Thereby we identify the contributions of the parent ion potential, and light induced sub-optical cycle electron dynamics on the observable energy and angular distributions.
\end{abstract}

(Some figures may appear in colour only in the online journal)

The basic principles of attosecond science in intense laser fields are readily understood with a few simplifications, such as treating the optical field classically, neglecting the parent ion potential after ionization and ignoring the detailed electronic structure of the parent atom or molecule. The last two decades have provided ample opportunity to probe the validity of these basic assumptions. In particular, the importance of the parent ion's Coulomb potential for the continuum electron wave packet has been widely recognized and studied in the ionization of atoms by linearly polarized light (e.g., [1-8]), where it has been named Coulomb focusing, and elliptically polarized light (e.g., [9-14]), where it is known as Coulomb asymmetry due to mainly a rotation of the photo-electron

\footnotetext{
${ }^{6}$ Present address: Novaled AG, Tatzberg 49, D-01307 Dresden, Germany. ${ }^{7}$ Present address: Carl Zeiss Microscopy GmbH, Rudolf-Eber-Str. 2, D-73447 Oberkochen, Germany.
}

angular distributions. The recently introduced technique of attosecond angular streaking relies on a precise accounting of this rotation that accompanies ionization by circular fields $[15,16]$. However, under some circumstances, the parent ion's potential can be neglected. For example, experiments along the lines of laser scanning tunnelling microscopy (laser-STM) [17] with the goal of imaging static [17-22] and transient $[23,24]$ electronic structure have largely ignored Coulomb effects. The extension of laser-STM to more complex systems, such as the recently suggested charge migration processes in molecular ions $[25,26]$, require a careful understanding of its limitations. While the influence of the Coulomb potential in atomic ionization is now well understood (e.g., [5, 27-32] and references therein), only a few attempts [33-37] have been made to unravel the qualitative effects on the strongfield photo-electron spectrum due to higher charge states and 
non-isotropic Coulomb potentials, i.e. distributed charges, as they occur in molecules.

Here, we study the effect of a doubly charged, twocentre Coulomb potential on the asymptotic momentum distribution of a tunnel-ionized photo-electron. For this purpose we recorded the strong-field-molecular-frame photoelectron angular distributions (SF-MFPADs) from doubly ionized $\mathrm{H}_{2}$ and $\mathrm{D}_{2}$ in circularly polarized, $800 \mathrm{~nm}$ laser pulses. The SF-MFPADs of the second ionization exhibit a very strong Coulomb asymmetry compared to the first ionization step. In addition they provide evidence for sub-cycle ionization bursts. We then compare the experiment to solutions of a reduceddimensionality time-dependent Schrödinger equation (TDSE). In order to clearly isolate the effect of the Coulomb potential on the photo-electrons, we couple the laser-dressed boundstate dynamics to a Coulomb-free continuum. We find that the molecular ion distorts and blurs the asymptotic momentum space of the photo-electron beyond a simple rotation of the SF-MFPADs.

Much of the complexity of laser-molecule interaction is already manifest in the hydrogen molecule. Hence, studies of $\mathrm{H}_{2}$ in intense laser pulses provide understanding of how the electronic structure of a molecule can give rise to new phenomena, such as electron localization-a fundamental response of molecular systems driven by strong laser fields. Electron localization is the underlying mechanism of phenomena such as bondsoftening [38, 39], charge resonance enhanced ionization (CREI) (thereafter referred to as enhanced ionization (EI)) [40], and sub-cycle ionization bursts [41] (for a review see, e.g. [42]). The so-called EI [40] is a sequential double ionization mechanism where the second ionization step is preceded by bondsoftening, a non-ionizing, light induced breaking of the molecular bond [38, 39]. The ionization then occurs typically at two to four times the equilibrium internuclear separation. While predicted for the neutral $\mathrm{H}_{2}$ [43], the overwhelming majority of experiments has associated EI with $\mathrm{H}_{2}^{+}$and other molecular ions as the precursor molecule (e.g., [44-52]). Molecular ions undergoing EI are therefore ideal candidates to study charge state and charge distribution effects on the ionized electron wave packet.

The experiment was performed using cold target recoil ion momentum spectroscopy [53]. The details of the experiment have been published before [50]. We use relatively low electrostatic extraction fields of $20-30 \mathrm{~V} \mathrm{~cm}^{-1}$ in our spectrometer. A Ti:Sa based regenerative amplifier was used to produce $40 \mathrm{fs}, 800 \mathrm{~nm}$ pulses, which were focussed to peak intensities of up to $3 \times 10^{14} \mathrm{~W} \mathrm{~cm}^{-2}$. For a subset of the data presented here, we used laser pulses from a second Ti:Sa laser system, which were spectrally broadened in an argon filled hollow core fibre and subsequently compressed to $10 \mathrm{fs}$ using chirped mirrors. Whereas in [50] we presented the ion energy spectra we now concentrate on the correlated electron spectra for circularly polarized light.

Figure 1(a) shows the kinetic energy release (KER) spectrum of the detected protons in the bondsoftening $\left(\mathrm{H}+\mathrm{H}^{+}\right)$and the EI channel $\left(\mathrm{H}^{+}+\mathrm{H}^{+}\right)$as a white area and a grey area, respectively. Since the bondsoftening channel yields only one charged fragment, the KER is calculated from twice the momentum of the detected proton. For the EI channel two correlated protons are required and their relative momentum is used to calculate the KER. The two peaks in the bondsoftening channel arise from dissociation via the net-1 and net- 2 photon channels $[38,39]$. Their individual width is dictated by the width of the nonadiabatic curve crossings of the field-coupled $1 \mathrm{~s} \sigma_{g}$ and $2 \mathrm{p} \sigma_{u}$ states in $\mathrm{H}_{2}^{+}$. Since the dissociation occurs after the first ionization, both bondsoftening channels are integrated for studying electron spectra. The structure in the KER spectrum of EI has been shown to arise from the interference of the nuclear wave packets in the net- 1 and net- 2 photon channels $[50,54]$ and has a related origin as the subcycle ionization bursts identified recently [41]. In contrast to bondsoftening, the KER spread for EI can be related to an internuclear distance $R$ at the instant of the second ionization step through the established relation $R=(\operatorname{KER}-1 \mathrm{eV})^{-1}$ $[55,56]$. Hence, we can compare the influence of singly and doubly charged Coulomb potentials as well as the effect of increasing anisotropy in the charge distribution.

In the following we make the assumption that the photo-electron emitted in the single ionization channel with subsequent dissociation (SI+BS channel), is identical to the primary ionization step in CREI, the double ionization channel of $\mathrm{H}_{2}$. This assumption is based on the well established sequential nature of CREI [44-50]. Although we can detect more than one electron, we cannot distinguish between the photo-electrons from the first and second ionization step on an event-by-event basis. Thus, the momentum distribution of the detected photo-electron in the CREI channel is an incoherent sum of a primary ionization and the secondary ionization. With the above assumption we can isolate the distributions pertaining to the secondary ionization by subtracting the distribution measured in the SI+BS channel.

In figure 1(b) we compare the electron energy spectra for the primary ionization step (white area) and the secondary ionization (grey area). The energy spectrum for the secondary ionization was obtained by subtracting the distribution for the primary ionization scaled to one half of the area in the EI channel. In circularly polarized light the asymptotic electron momentum describes a torus in the polarization plane, i.e. the electron energy will be proportional to the peak intensity [57, 58]. Furthermore, in the absence of the ion potential, the asymptotic electron momentum can be shown to point at an angle of $90^{\circ}$ relative to the field at the time of ionization [19-21, 23].

Although we measure the electron distribution in the lab frame from an unaligned ensemble of $\mathrm{H}_{2}$ molecules, the detection of a proton fixes the molecular axis and allows the transformation ex post facto into the molecular frame. Thereby we implicitly assume that the dissociation occurs fast on the time scale of rotation and is known as the axialrecoil approximation. Figure 1(c) shows the molecular-frame photo-electron angular distribution from the first ionization step, followed by the breakup of $\mathrm{H}_{2}^{+}$via bondsoftening (white area), and the second ionization step (grey area), followed by Coulomb explosion. In the latter SF-MFPAD we removed the contribution of the first ionization step by subtracting the SF-MFPAD of the bondsoftening channel. 
(a)

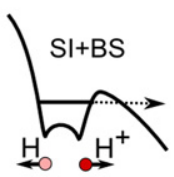

(b)
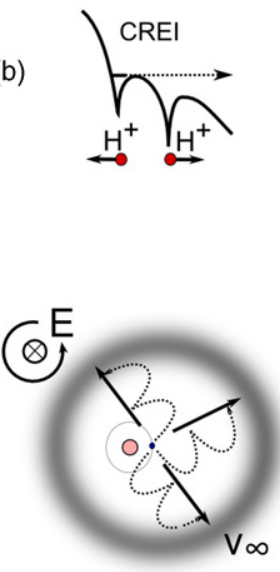

(c)

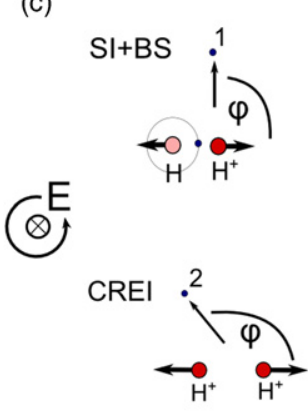

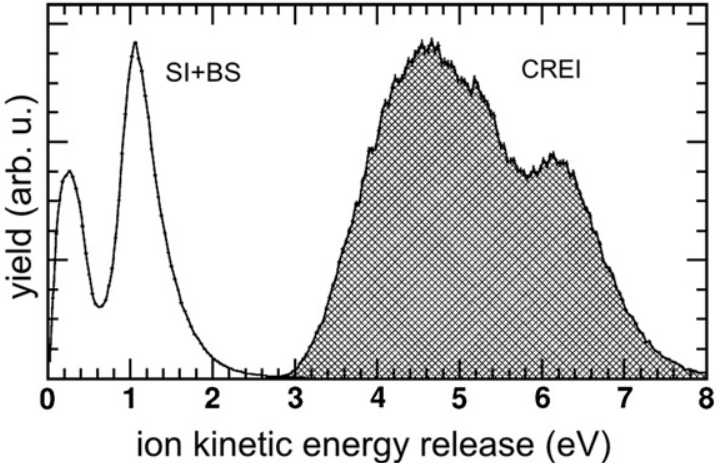
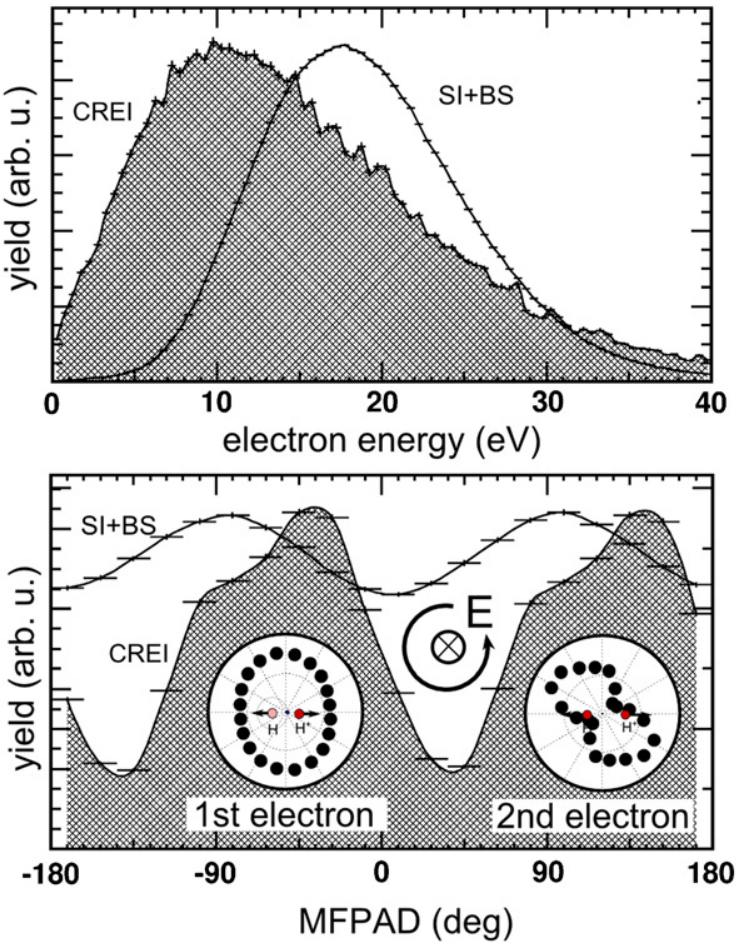

Figure 1. Concept and basic spectra for ionization of $\mathrm{H}_{2}$ molecules subjected to circularly polarized, $800 \mathrm{~nm}, 40 \mathrm{fs}$ pulses at a peak intensity of $3 \times 10^{14} \mathrm{~W} \mathrm{~cm}^{-2}$. (a) (Left) Illustration of single ionization followed by bondsoftening (SI+BS) and CREI of the dissociating molecular ion (bottom). (Right) KER spectrum of SI+BS (empty area) and CREI (grey area). Both channels are drawn on different vertical scales. (b) (Left) In circular light, observable electron momenta are describing a torus that lies in the plane of polarization, and are driven away from the ion perpendicular to the direction of tunnelling. (Right) Electron energy spectra associated with the first (empty area) and the second (grey area) ionization step in EI. (c) (Left) Illustration, how the SF-MFPAD is obtained from the detected proton and electron momentum. (Right) SF-MFPADs of the bondsoftening (empty area) and EI (grey area) integrated over photo-electron energy and KER. Shown is also the helicity of the laser polarization.

The angular ionization probability of the neutral molecule peaks at $\approx 95^{\circ}$. Hence, for studies of static electronic structure of neutrals using circular light (e.g., [19, 20]), the $90^{\circ}$ rule is a good approximation. However, further ionization from the molecular ion is prone to significant distortions of the asymptotic momentum. The angular distributions in the EI channel bears a large resemblance to SF-MFPADs of tunnel ionization from atoms in elliptical light (e.g., [59]). The inset in figure 1(c) shows the SF-MFPADs in polar coordinates. In figure 1 the angular distributions were integrated over all proton and electron energies. In the following we will resolve the SF-MFPADs for proton and electron energy.

When the polarization of the laser light is inverted, the rotation of the SF-MFPADs visible in figure 1(c) is reversed. This is in correspondence to circular dichroism in single photon ionization (e.g., [60-62]), where the deviation from the fourfold symmetry of MFPADs is inverted when the helicity of the photon changes sign. Hence, Coulomb asymmetry can be seen as a multi-photon version of circular dichroism: our MFPADs turn the other way if we use light with opposite polarization (see also [10]).

In figure 2(a) we plot the triple coincidence energy map of two detected protons and one detected photo electron for EI of $\mathrm{H}_{2}$ at peak intensities of $I=2 \times 10^{14} \mathrm{~W} \mathrm{~cm}^{-2}$ and $I=3 \times 10^{14} \mathrm{~W} \mathrm{~cm}^{-2}$ and, for the higher intensity, for molecular deuterium $\mathrm{D}_{2}$. As shown in detail elsewhere [50] one can clearly identify the effects these parameter variations have: the lower intensity results in a lower average electron energy, as predicted by simple models [58]. For the heavier isotope of the molecule the slower nuclear dynamics results in a 

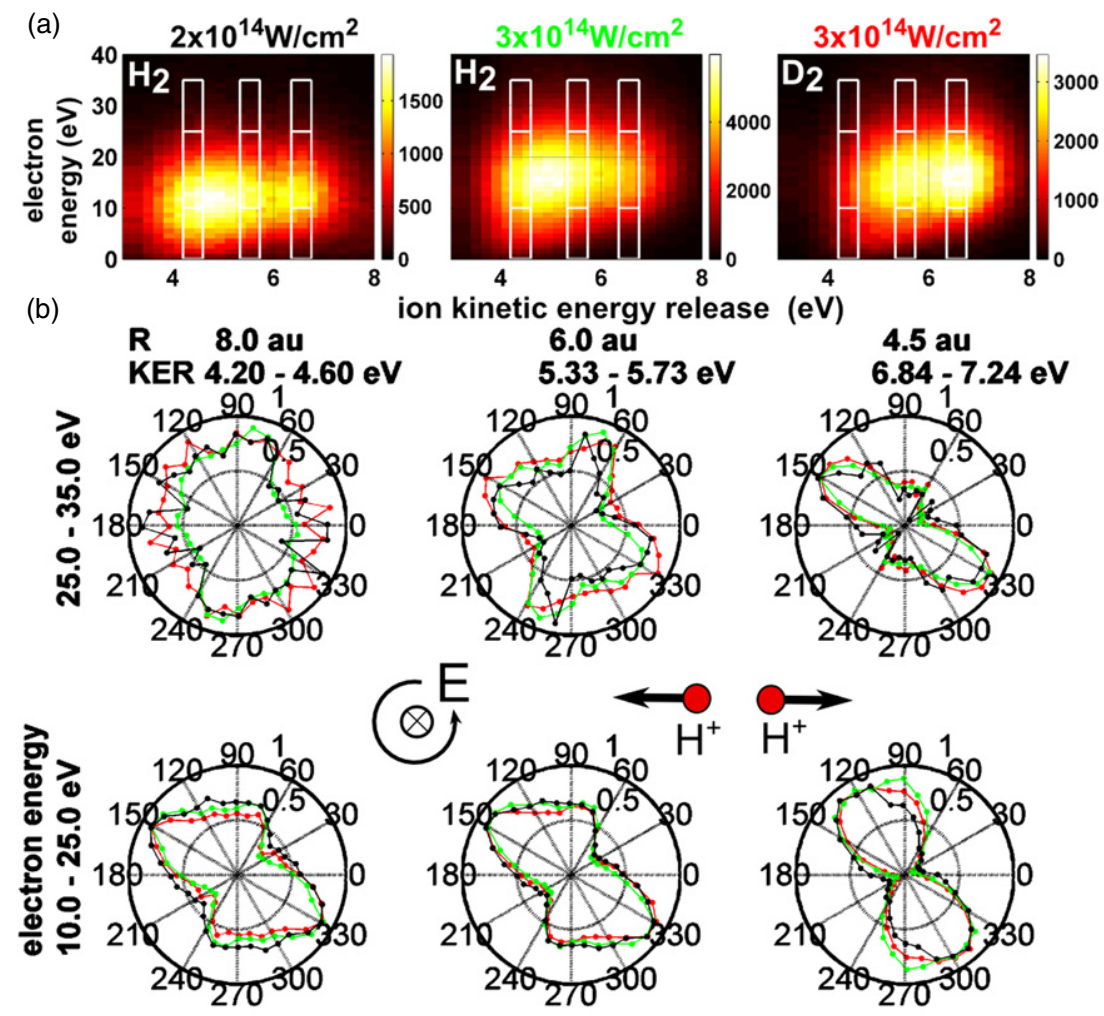

(1)
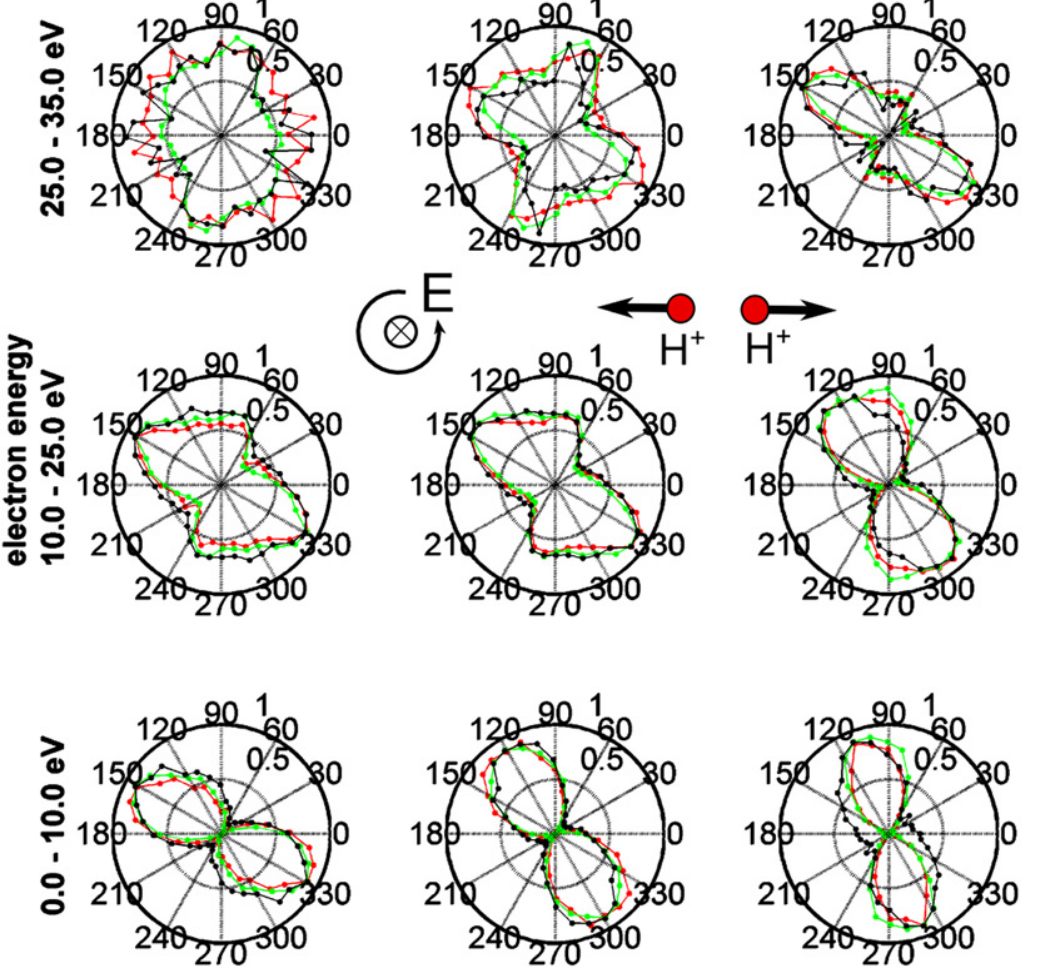

Figure 2. (a) Triple coincidence energy maps of two protons and photo-electron for $\mathrm{H}_{2}$ and $\mathrm{D}_{2}$, different intensities in $40 \mathrm{fs}, 800 \mathrm{~nm}$ laser pulses. (b) Polar plots of photo-electron angular distributions in the molecular frame as a function of electron energy and KER as indicated by boxes in (a). The angular distribution of the first electron is subtracted (see text). The two intensities and the $\mathrm{D}_{2}$ data set are colour coded according to the intensity labels in (a). Connecting line to guide the eye. The data in each polar plot has been normalized to unity in order to facilitate the comparison of angular features. The indicated internuclear distance is calculated according to $R=(\mathrm{KER}-1 \mathrm{eV})^{-1}[55,56]$. The molecular axis and the handedness of the light polarization is indicated.

redistribution of the nuclear energies to higher values, while the photo-electron energy distribution remains unaffected. Since the KER is directly related to the internuclear separation at the time of the second ionization, the isotopical effect can be attributed to the finite pulse envelope.

However, one feature of EI evidenced in these histograms cannot be explained within a simple, Coulomb-free picture of the ionization mechanism. Photo-electron energies extending down to almost zero energy not only lack a description within the so-called simple man's model of tunnel ionization in a circularly polarized laser field [63], but they are also not encountered in experiments, with a few exceptions [64, 34]. Figure 2(a) shows, that the low energy electrons are prevalent mainly for the lower KER, i.e. larger internuclear separations. For higher KER the low energy electrons gradually recede. In contrast, the high-energy side of the photo-electron appears independent of KER. Finally, the white boxes drawn in the energy maps are indicating subsets of the data that we will use in the following to study the associated SF-MFPADs.

In figure 2(b) the SF-MFPADs for the subsets of data indicated in (a) are shown. Here, $0^{\circ}$ correspond to a photoelectron emission parallel to the first detected proton. As in figure 1(c) we removed the angular distribution of the primary electron. Each angular distribution was normalized to the angle with the maximum counts. Thereby the information about the relative cross section, i.e. the number of detected electrons in the subsets, is traded for an improved comparability of the angular distributions.

The lowest energy electrons $(<10 \mathrm{eV})$ are produced exclusively in the EI step, see figure 1(b) whereas the angular distributions at energies $>10 \mathrm{eV}$ are at least partially contaminated by the primary ionization step. Comparison of the different data sets reveals that, within the experimental resolution, the SF-MFPADs are independent of intensity or the nuclear dynamics. Hence, the breakdown of the fourfold 
symmetry relative to the molecular axis can only have two origins: the Coulomb potential, or pre-ionization, sub-optical cycle, bound electron dynamics.

Previously, there has been a discussion of the influence of Coulomb effects in the continuum propagation, as well as the influence of the sub-cycle ionization bursts on the SF-MFPADs in $\mathrm{EI}$ of $\mathrm{H}_{2}^{+}[41,34]$. In order to assess these contributions, we have constructed a frozen-nuclei TDSE method that allows one to include or exclude Coulomb effects on the continuum as well as include the bound-state dynamics responsible for multiple ionization bursts. When the Coulomb field is excluded this method reduces to a TDSE version of the strong-field approximation.

We use a two-dimensional two-centre soft-core model. In atomic units $\left(m_{e}=\hbar=e=1\right)$ the laser+system Hamiltonian is

$$
\begin{gathered}
H(x, y, t)=-\frac{1}{2} \nabla^{2}-\frac{1}{\sqrt{(x \pm R / 2)^{2}+y^{2}+0.8}} \\
-\frac{1}{\sqrt{(x+R / 2)^{2}+y^{2}+0.8}}+x E_{x}(t)+y E_{y}(t)
\end{gathered}
$$

where $R$ is the bond length and $E(t)$ the laser electric field

$$
\vec{E}(t)=-\frac{\partial}{\partial t} \vec{A}(t)
$$

with the vector potential $\vec{A}(t)$ chosen to be

$$
\vec{A}(t)=\frac{E_{0}}{\omega} f(t)[-\sin (\omega t) \hat{x}+\cos (\omega t) \hat{y}] .
$$

The envelope function $f(t)$ is

$$
f(t)= \begin{cases}0, & t \leqslant 0 \\ \sin ^{2}\left(\frac{\pi t}{2 \tau}\right), & 0<t \leqslant 2 \tau \\ 0, & t>2 \tau\end{cases}
$$

and $\tau=400 \mathrm{au}$ is the full-width at half-maximum of the electric field, corresponding to a FWHM in intensity of $7 \mathrm{fs}$.

The photo-electron spectrum that results from the interaction of the laser pulse with the model system is calculated by solving the TDSE numerically using the Fourier split-operator method on a grid of \pm 1000 au with 4096 points along each direction. This grid is large enough to avoid collisions between the continuum wave packet and the grid edge. The system is initialized in the ground state and the Schrödinger equation is integrated in time over the duration of the pulse. The time step used in the simulations is $\Delta t=$ $0.05 \mathrm{au}$ At the end of the pulse, we apply a mask function to the final wavefunction $\Psi_{f}(x, y)$ to remove the core states (and any low energy continuum portions that are still located near the binding potential):

$$
\Psi_{f}^{\prime}(x, y)=M(x, y) \Psi_{f}(x, y)
$$

where the mask is given by

$$
M(x, y)=\operatorname{erf}\left(\frac{\sqrt{x^{2}+y^{2}}-25}{5}\right)+0.5 .
$$

The final photoelectron momentum spectrum is then given by the spatial Fourier transform of the masked wavefunction

$$
S\left(p_{x}, p_{y}\right)=\left|\iint \Psi_{f}^{\prime}(x, y) \mathrm{e}^{\mathrm{i}\left(p_{x} x+p_{y} y\right)} \mathrm{d} x \mathrm{~d} y\right|^{2} .
$$

In figure 3 we compare the SF-MFPAD obtained from our simulations with experimental data obtained with $40 \mathrm{fs}$, $3 \times 10^{14} \mathrm{~W} \mathrm{~cm}^{-2}$ laser pulses and data obtained with $10 \mathrm{fs}$, $25 \times 10^{14} \mathrm{~W} \mathrm{~cm}^{-2}$ laser pulses. Figure 3(a) shows the energy maps of the experimental data sets and indicates the subsets to be compared with the simulation. The change of scale in comparison to figure 2(a) was necessary to accommodate the much larger energy spread for the $10 \mathrm{fs}$ pulses.

In figure 3(b) we plot the subsets of (a) indicated by the white boxes in polar coordinates. Note, that the subsets have changed for the 40 fs data compared to figure 2 . The solid, red lines in figure 3(b) show the calculated $S\left(p_{x}, p_{y}\right)$ in polar coordinates for several bond lengths. The bond lengths have been approximately matched to the KER ranges of the experimental data. In the simulations we have averaged the photo-electron spectra over a range of intensities to minimize the strong appearance of ATI rings. Specifically, averaging was done over peak intensities of $1.66-3.0 \times 10^{14} \mathrm{~W} \mathrm{~cm}^{-2}$ using 20 points, all uniformly weighted. Although the simulation averages over a significant intensity range, the intensity independence of the SF-MFPADs in figure 2 suggests that the angular distributions can be compared for intensities varying within a factor of two. Further, since the model is a simple twodimensional, two-centre model, we do not expect a one-to-one correspondence between theory and experiment, and it thus makes sense to compare qualitative features.

For the range of electron energies $<15 \mathrm{eV}$ (bottom row), the SF-MFPADs are well described by a simple $\sin ^{2}\left(\phi-\phi_{0}\right)$ like distribution, with the alignment angle of the distribution $\phi_{0}$ being the main characteristic for the varying KER. In particular, the angle $\phi_{0}$ increases from $\approx 10^{\circ}$ to $\approx 60^{\circ}$ as the internuclear separation increases from $R=2$ au to $R=8$ au. In this range of electron energies the simulation is in very good agreement with both experimental data sets for all internuclear separations despite the large range in the experimental parameters, manifest in the very different correlated electron energy-KER spectra (figure 2(a)).

At a tenfold higher intensity Coulomb effects on the photo-electron are greatly reduced, since the corresponding semiclassical trajectories have a threefold larger excursion length. Thus, the fact that the experimental SF-MFPADs agree, suggests that Coulomb asymmetry arises near the tunnel exit, i.e. the instant the electron wavepacket is leaving the ion. Even at electron energies $>15 \mathrm{eV}$ a significant rotation of the SF-MFPAD is observed for the high intensity data. This observation supports the mechanism proposed in [34] and contradicts the model that attributes Coulomb asymmetry to the first turning point of the ionized electron, far away from the parent ion [13].

A more complex angular structure appears at electron energies above $>15 \mathrm{eV}$ for both experiment and theory. For smaller internuclear separations the simulation reproduces the low intensity experiment very well. However, at large internuclear separations, the complexity of the theoretical SFMFPADs cannot be found in the experimental data. A possible explanation for this discrepancy is the experimental averaging. In the experiment a range of isointensity shells are present in the focus, leading to an averaging of intensity dependent 


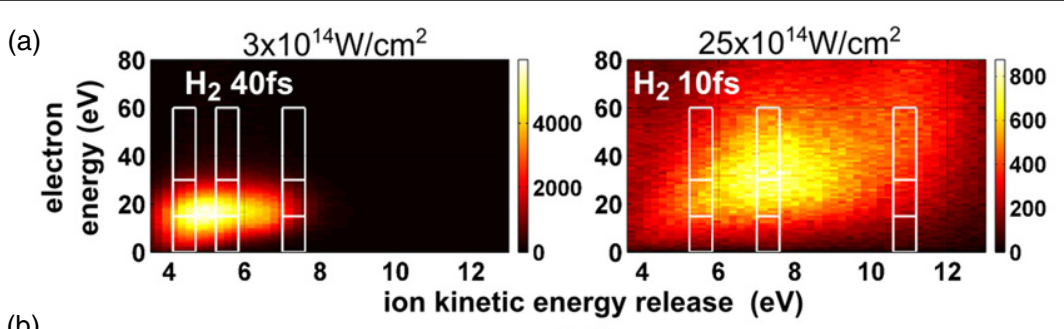

(b)
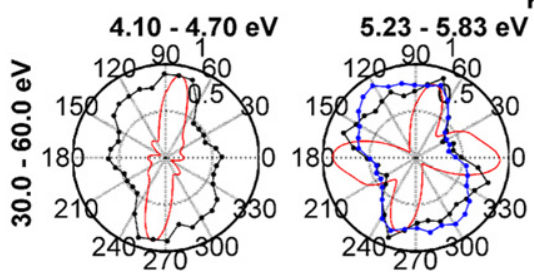

KER
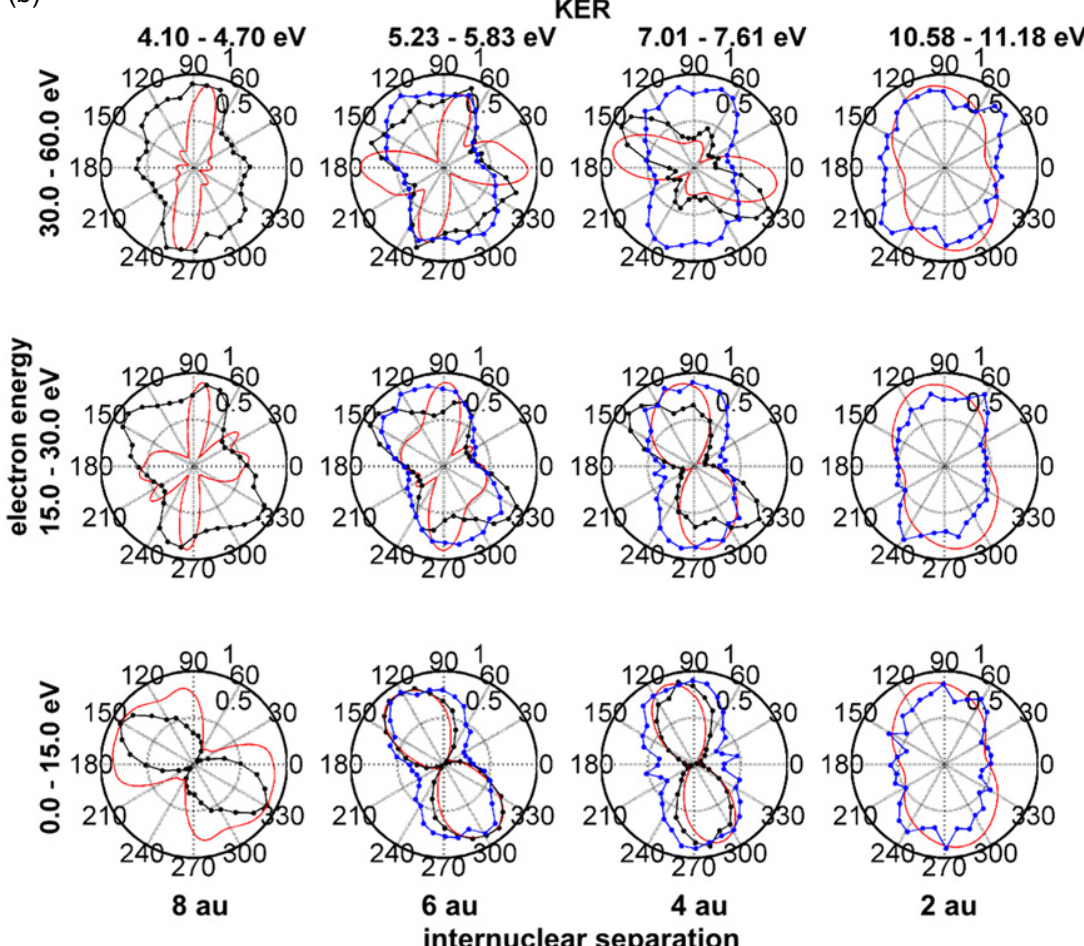

Figure 3. (a) Experimental triple coincidence energy maps of two protons and one photo-electron from double ionization of $\mathrm{H}_{2}$ with $40 \mathrm{fs}$, $3 \times 10^{14} \mathrm{~W} \mathrm{~cm}^{-2}$ (left) and $10 \mathrm{fs}, 25 \times 10^{14} \mathrm{~W} \mathrm{~cm}^{-2}$ (right) pulses. (b) Polar plots of SF-MFPADs as a function of electron energy and KER as indicated by boxes in (a). The subsets for the low intensity (black dots) and high intensity (blue dots) data overlap for their highest and lowest KER regions, respectively. (The low intensity data were shown in figure 2 but with different slices in electron energy.) Solid, red line: results of a TDSE simulation of $\mathrm{H}_{2}^{+}$with fixed nuclei in a $7 \mathrm{fs}, 1.66-3.0 \times 10^{14} \mathrm{~W} \mathrm{~cm}^{-2}$ pulse (see text for details). The electric field used in the simulation has the same helicity as the experiment. The internuclear distance used in the simulations is indicated at the bottom of (b).

effects. This intensity averaging effect is more pronounced for the experiment at the higher peak intensity, supported by the much simpler angular distributions in the high intensity data. The frozen nuclei approximation in the simulation cannot explain the lack of structure in the experiment, since the SFMFPADs for $\mathrm{H}_{2}$ and $\mathrm{D}_{2}$ are identical (compare figure 2). Finally, a pulse envelope effect cannot be responsible, due to the comparative lack of angular structure in the experiment with 10 fs pulses.

At the smallest internuclear distance $(R=2 \mathrm{au}, K E R=$ $10.58-11.18 \mathrm{eV}$ ) simulation and experiment resemble the structureless, slightly rotated, single ionization SF-MFPAD. This observation does not contradict the concept of multiple ionization bursts, since their origin, as suggested by Takemoto et al [41], is electron localization through charge resonant states at internuclear separations $>4$ au.

In figure 4 the molecular-frame emission angle as a function of the photo-electron energy is shown in twodimensional histograms, for both, experiment (a) and theory (b), and for three different KER ranges and their corresponding internuclear distances (1-3). The experimental data shown are for $40 \mathrm{fs}, 800 \mathrm{~nm}, 3 \times 10^{14} \mathrm{~W} \mathrm{~cm}^{-2}$ laser pulses, the theory data are underlying the theoretical SF-MFPADs in figure 3. Remarkably, the simulation yields higher (a1, b1) as well as lower (a3, b3) electron energies than the experiment. Furthermore, the fine structure of the simulated SF-MFPADs at higher photo-electron energies visible in (b2, b3) is not reflected by the experiment. It is this angular structure that has been associated with sub-cycle multiple ionization bursts [34]. The lack of fine structure in the experimental data cannot be fully explained by a smaller dynamic range in the color scale for the experiment, but it is likely due to the nuclear dynamics in the experiment and the associated angular averaging.

In the following we will describe our approach to modify the simulation in order to isolate the parent ion's potential at will and to identify the origin of the structure in the SF-MFPADs. First, the full Schrödinger equation is solved as previously described to obtain the full timedependent wavefunction $\Psi(x, y, t)$. The full wavefunction 

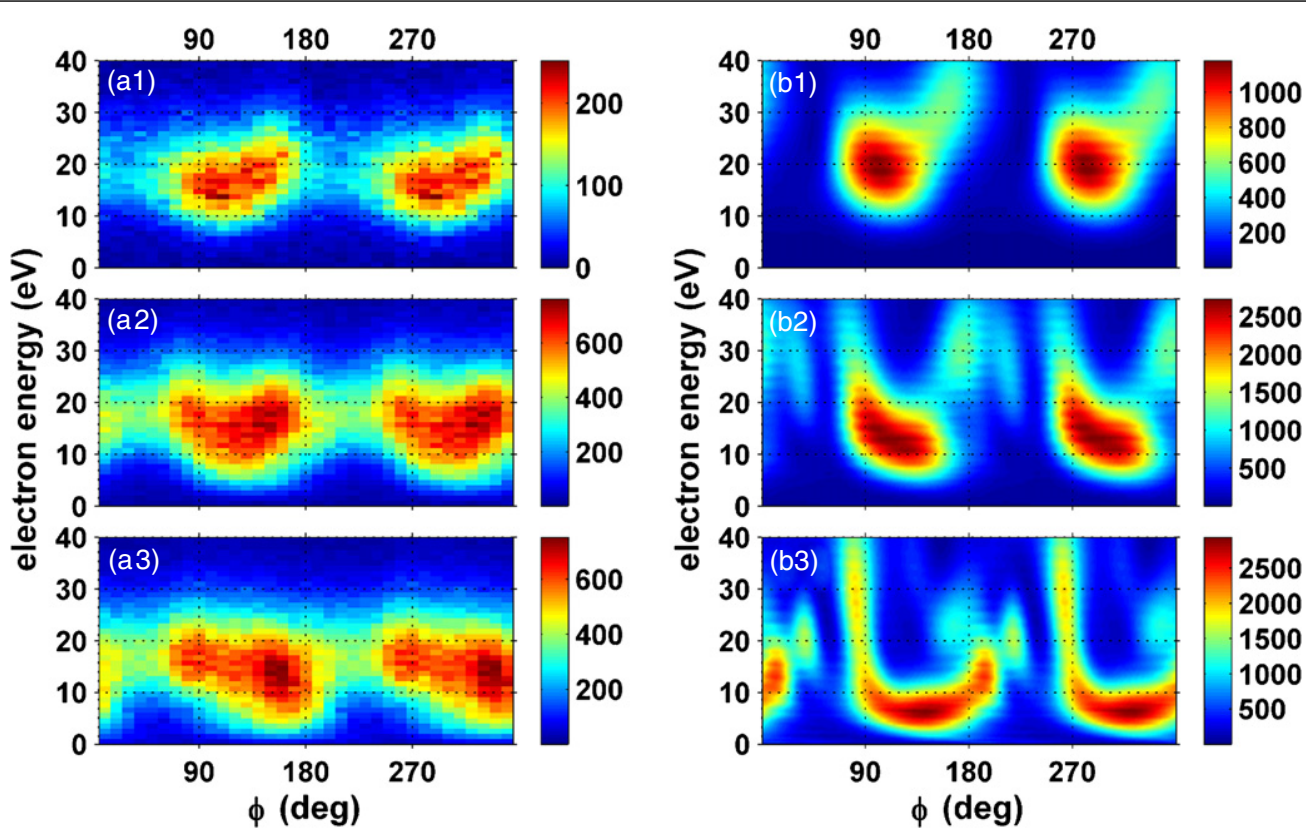

Figure 4. Photo-electron distributions of $\mathrm{H}_{2}^{+} \mathrm{EI}$ in polar coordinates for (a) experiment at $40 \mathrm{fs}, 3 \times 10^{14} \mathrm{~W} \mathrm{~cm}^{-2}$ and (b) theory using a $7 \mathrm{fs}$, $1.66-3.0 \times 10^{14} \mathrm{~W} \mathrm{~cm}^{-2}$ pulse. (a1) KER: 7.01-7.61 eV, (a2) KER: 5.23-5.83 eV, (a3) KER: 4.10-4.70 eV. (b1) R: 4 au, (b2) R: 6 au, (b3) R: 8 au.

is then projected onto the low-lying field-free bound states $\varphi_{i}(x, y)$

$$
c_{i}(t)=\iint \varphi_{i}^{*}(x, y) \Psi(x, y, t) \mathrm{d} x \mathrm{~d} y
$$

to extract the field-driven bound-state dynamics. The timedependent bound-state portion of the full wavefunction can then be reconstructed by summing over a truncated set of bound states

$$
\Psi_{B}(x, y, t)=\sum_{i=0}^{N} c_{i}(t) \varphi_{i}(x, y)
$$

where $N$ is a small number that truncates the number of bound states used. For the present two-centre system, the main boundstate dynamics is field-driven charge oscillations between the two centres. The ground state and first excited state are together adequate to capture most features of these charge oscillations.

Once the bound-state dynamics has been correctly captured by $\Psi_{B}(x, y, t)$, we proceed to couple this to a 'flat' continuum (i.e. no Coulomb or binding potential effects)

$\mathrm{i} \frac{\partial}{\partial t} \Psi_{c}(x, y, t)=\left[-\frac{1}{2} \nabla^{2}+x E_{x}(t)+y E_{y}(t)\right] \Psi_{c}(x, y, t)$

where $\Psi_{B}(x, y, t)$ acts as a source term that populates the continuum $\Psi_{c}(x, y, t)$, which has the initial condition $\Psi_{c}(x, y, t=0)=0$. Although we are implementing specifically equation (10), it should be noted that this equation can be recast in integral form to give

$$
\begin{aligned}
& \Psi_{c}(x, y, t)=\mathrm{i} \int \mathrm{d} \vec{p} \int_{0}^{t} \mathrm{~d} t^{\prime} \phi_{\vec{p}}(x, y, t) \\
& \times \exp \left[-\frac{\mathrm{i}}{2} \int_{t^{\prime}}^{t}|\vec{p}+\vec{A}(\tau)|^{2} \mathrm{~d} \tau\right] A_{I}(\vec{p}, t)
\end{aligned}
$$

where

$$
A_{I}(\vec{p}, t)=\int \phi_{\vec{p}}^{*}(x, y, t)\left[E_{x}(t) x+E_{y}(t) y\right] \Psi_{B}(x, y, t) \mathrm{d} x \mathrm{~d} y
$$

and

$$
\phi_{\vec{p}}(x, y, t)=(2 \pi)^{(3 / 2)} \mathrm{e}^{\mathrm{i}[\vec{p}+\vec{A}(t)] \cdot \vec{r}} .
$$

Equations (11) can now be recognized as SFA equations coupling the dressed bound-state wave packet $\Psi_{B}(x, y, t)$ to a flat (i.e. plane wave) continuum $\Psi_{c}(x, y, t)$.

The mechanism of multiple sub-cycle ionization bursts is based on the idea that bound-state dynamics creates charge oscillations between the two molecular centres, and that these charge oscillations can shift the moment of ionization relative to the peak of the field. In particular, the model predicts multiple ionization bursts of electrons with significant initial momentum, when the bound-state wave packet shows multiple charge oscillations within a single halfcycle [34]. We test this idea using the above approach which removes Coulomb effects from the continuum. For the sake of clarity we will not use the experimental intensity, where multiple sub-cycle ionization bursts can be assumed. Instead we will consider a single peak intensity of $1.5 \times 10^{14} \mathrm{~W} \mathrm{~cm}^{-2}$, disregarding spatial intensity averaging, where only a single sub-cycle ionization burst will be present.

In order to track the bound-state charge oscillations, in figure 5(a) we plot the bound-state dipole $\iint\left|\Psi_{B}(x, y, t)\right|^{2} x \mathrm{~d} x \mathrm{~d} y$ that lies along the molecular axis. The internuclear distance is fixed to 4 au. Sub-cycle charge oscillations can be seen. In particular, one of the charge oscillations starts to lag the peak of the field by about $24^{\circ}$ (see solid red vertical lines in figure 5(a)) while the second oscillation is shifted too close to the point of zero field to 

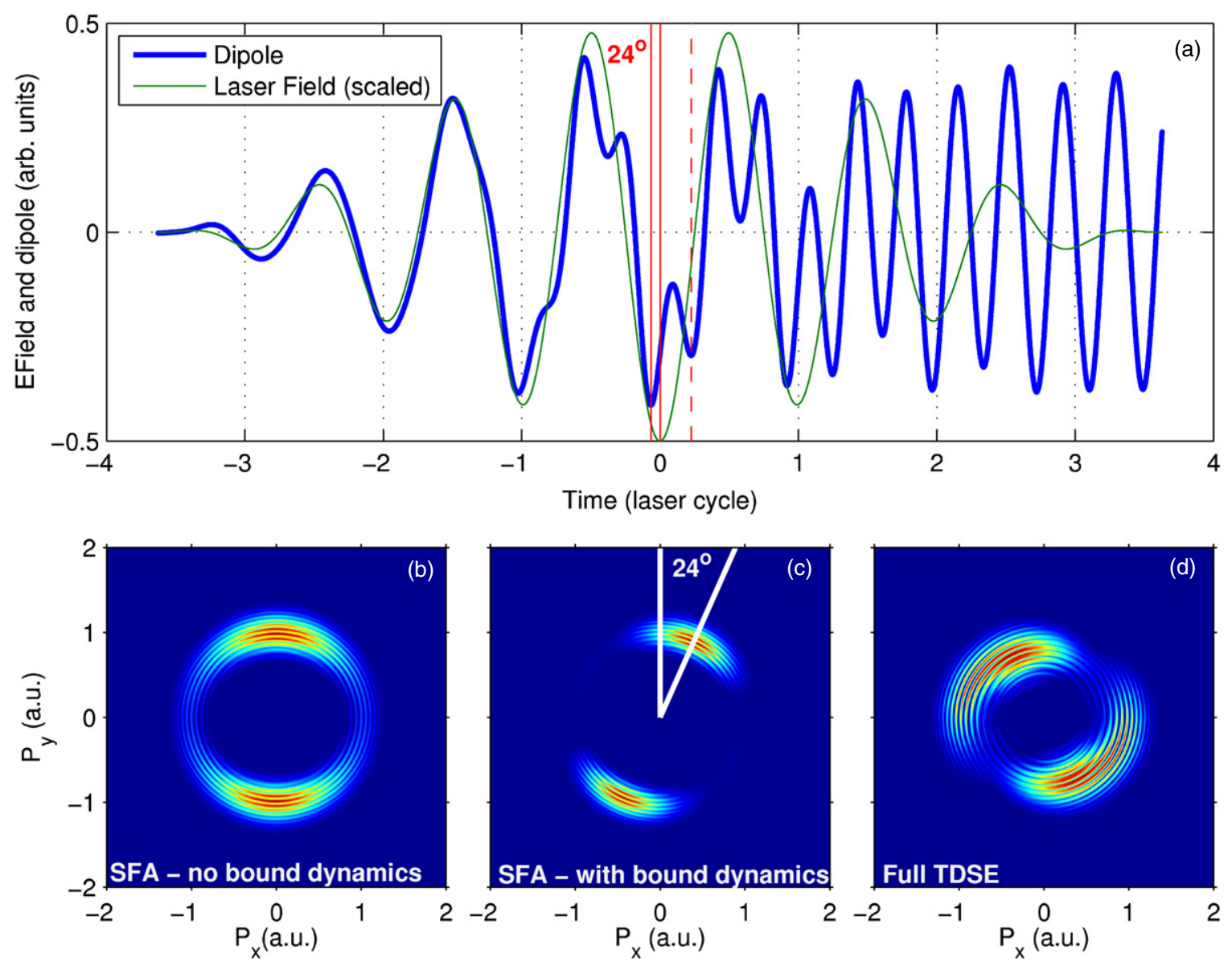

Figure 5. (a) Laser field component along the molecular axis, corresponding to a peak intensity of $1.5 \times 10^{14} \mathrm{~W} \mathrm{~cm}^{-2}$ in the circularly polarized field, and $\mathrm{H}_{2}^{+}$calculated dipole moment at an internuclear separation of 4 au. (b) Asymptotic momentum photo-electron spectrum for the field-free groundstate coupled to the flat continuum (i.e. traditional SFA). Molecular axis along $x$, electric field rotating counterclockwise (c) The laser-driven bound-state wave packet coupled to flat, i.e. plane wave continuum. (d) as (c) but coupled to a full Coulomb continuum.

contribute significantly to ionization (see dashed vertical red line in figure 5(a)).

In figure 5(b) we show the photo-electron momentum spectrum for an undressed bound state coupled to the flat continuum, i.e. $\Psi_{B}(x, y, t)=\varphi_{0}(x, y) \mathrm{e}^{-\mathrm{i} E_{0} t}$, where $\varphi_{0}(x, y)$ and $E_{0}$ are the field-free ground state and energy. In the figure the molecular axis is horizontal and the light polarization rotates counter-clockwise. In this case, which is the traditional SFA result, the final electron momentum peaks along $90^{\circ}$ with respect to the molecular axis, as expected.

In figure 5(c) we show the photo-electron spectrum that results from coupling the laser-driven bound-state wave packet to the flat (i.e. no Coulomb effects) continuum. The distribution exhibits a rotation of $-24^{\circ}$ relative to the results without bound-state dynamics in figure 5(b). The observed rotation coincides exactly with the shift of the dipole oscillations relative to the field peak seen in figure 5(a), indicating that bound-state dynamics can indeed influence angular shifts and structures in the final photo-electron spectrum. The second peak in the dipole oscillations that appears near $E(t)=0$ is much less pronounced in the photo-electron spectra since the ionization rate near $E(t)=0$ is very small.

Figure 5(d), finally, shows the photo-electron spectrum calculated using the full Schrödinger equation with full
Coulomb effects on the continuum. As can be seen by comparing figures 5(c) and (d), the 'Coulomb-free' model fails to capture the sense of rotation and the structure of the full simulation. The sub-cycle bound-state electron dynamics can lead to an ejection before the field maximum aligns with the molecular axis. Consequently the observable electron momentum appears at an angle less than $90^{\circ}$ to the molecular axis. It is important to realize, that due to figure 5(c) this observation can be explained without an initial momentum, although it does not contradict a previous analysis that invoked the specific direction of an initial momentum at the instant of tunnelling [34]. Without a Coulomb field the final momentum corresponds directly to the timing of the electron emission. However, when the Coulomb field is accounted for, the resulting angular structure cannot be related to an ionization time. In particular, the Coulomb potential results in a slowingdown of the photo-electron, i.e. the radial momentum is decreased, and a spreading and deflection of the angular distribution $>90^{\circ}$.

In conclusion, in order to study the applicability of the laser-STM concept to molecular ions, we have measured the SF-MFPADs of $\mathrm{H}_{2}$ double ionization in intense, near-infrared, circularly polarized laser pulses, resolved for both sequential ionization steps. The SF-MFPAD of the first ionization step, 
i.e. the ionization of the neutral molecule, deviates by a rotation of $5^{\circ}$ from a Coulomb-free model. On the other hand, the SF-MFPAD of the second ionization step, i.e. the ionization of $\mathrm{H}_{2}^{+}$, exhibits complex structure originating in the laser driven sub-optical cycle electron dynamics. The subcycle-nature of the second ionization is obscured by a strong Coulomb effect. We find that the SF-MFPADs are rotated with respect to a Coulomb-free model depending on the bond length at the time of ionization. This rotation is independent over a wide range of laser intensities, suggesting that Coulomb asymmetry arises at the exit of the tunnel [34] or through the scattering off a proton [35]. These results lead us to conclude that for imaging of electronic structure in molecular ions it is imperative to decrease the strong influence of the parent ion's Coulomb potential. This could be achieved by employing longer wavelength light.

Very recently it has been demonstrated that not only the long-range part of the Coulomb potential acting on the electron but also on the phase of the bound-state wavepacket upon ionization is imprinted in the electron spectra [32]. We have shown in this paper that for the hydrogen molecule, the situation is even more complex: the spectra can only be explained by a combination of bound electronic dynamics (which features different ionization dynamics depending on the internuclear distance), and the effect of the Coulomb potential acting on the ionized electron.

\section{Acknowledgments}

The experimental work is supported by the NRC HGF science \& technology fund, Austrian Science Fund FWF, grant V193N16, DFG and BMBF (Internationales Büro). We are indebted to $\mathrm{S}$ Patchkovskii for always enlightening discussions.

(c) NRC Canada 2012.

\section{References}

[1] Schwengelbeck U and Faisal F H M 1994 Ionization of the one-dimensional Coulomb atom in an intense laser field Phys. Rev. A 50632

[2] Brabec T, Ivanov M Y and Corkum P B 1996 Coulomb focusing in intense field atomic processes Phys. Rev. A 54 R2551

[3] Rudenko A, Zrost K, Ergler T, Voitkiv A B, Najjari B, de Jesus V L B, Feuerstein B, Schröter C D, Moshammer R and Ullrich J 2005 Coulomb singularity in the transverse momentum distribution for strong-field single ionization J. Phys. B: At. Mol. Opt. Phys. $\mathbf{3 8}$ L191

[4] Comtois D, Zeidler D, Pépin H, Kieffer J C, Villeneuve D M and Corkum P B 2005 Observation of Coulomb focusing in tunnelling ionization of noble gases J. Phys. B: At. Mol. Opt. Phys. 381923

[5] Abu-samha M, Dimitrovski D and Madsen L B 2008 The role of the atomic potential in the regime of strong-field tunnelling ionization: imprints on longitudinal and 2D momentum distributions J. Phys. B: At. Mol. Opt. Phys. 41245601

[6] Blaga C I, Catoire F, Colosimo P, Paulus G G, Muller H G, Agostini P and DiMauro L F 2009 Strong-field photoionization revisited Nature Phys. 5335

[7] Quan W et al 2009 Classical aspects in above-threshold ionization with a midinfrared strong laser field Phys. Rev. Lett. 103093001
[8] Gräfe S, Doose J and Burgdörfer J 2012 Quantum phase-space analysis of electronic rescattering dynamics in intense few-cycle laser fields J. Phys. B: At. Mol. Opt. Phys. 45055002

[9] Trombetta F, Ferrante G and Basile S 1988 J. Phys. B: At. Mol. Opt. Phys. 21 L539

[10] Bashkansky M, Bucksbaum P H and Schumacher D W 1988 Asymmetries in above-threshold ionization Phys. Rev. Lett. 602458

[11] Basile S, Ferrante G and Trombetta F 1988 Multiphoton multichannel ionisation of hydrogen with elliptical J. Phys. B: At. Mol. Opt. Phys. 21 L377

[12] Paulus G G, Grasbon F, Dreischuh A, Walther H, Kopold R and Becker W 2000 Above-threshold ionization by an elliptically polarized field: interplay between electronic quantum trajectories Phys. Rev. Lett. 843791

[13] Goreslavski S P, Paulus G G, Popruzhenko S V and Shvetsov-Shilovski N I 2004 Phys. Rev. Lett. 93233002

[14] Chu W et al 2012 Unexpected breakdown of the simple mans model for strong-field photoionization in the high-energy recollision region Phys. Rev. A 85021403

[15] Eckle P, Pfeiffer A N, Cirelli C, Staudte A, Dörner R, Muller H G, Büttiker M and Keller U 2008 Attosecond ionization and tunneling delay time measurements in helium Science 3221525

[16] Pfeiffer A N, Cirelli C, Smolarski M, Dimitrovski D, Abu-samha M, Madsen L B and Keller U 2012 Attoclock reveals natural coordinates of the laser-induced tunnelling current flow in atoms Nature Phys. 876

[17] Meckel M et al 2008 Laser induced electron tunneling and diffraction Science 3201478

[18] Pavičić D, Lee K F, Rayner D M, Corkum P B and Villeneuve D M 2007 Direct measurement of the angular dependence of ionization for $\mathrm{N}_{2}, \mathrm{O}_{2}$, and $\mathrm{CO}_{2}$ in intense laser fields Phys. Rev. Lett. 98243001

[19] Akagi H, Otobe T, Staudte A, Shiner A, Turner F, Dörner R, Villeneuve D M and Corkum P B 2009 Laser tunnel ionization from multiple orbitals in HCL Science 3251364

[20] Staudte A et al 2009 Angular tunneling ionization probability of fixed-in-space $\mathrm{H}_{2}$ molecules in intense laser pulses Phys. Rev. Lett. 102033004

[21] Magrakvelidze M, He F, De S, Bocharova I, Ray D, Thumm U and Litvinyuk I V 2009 Angular dependence of the strong-field ionization measured in randomly oriented hydrogen molecules Phys. Rev. A 79033408

[22] Holmegaard L et al 2010 Photoelectron angular distributions from strong-field ionization of oriented molecules Nature Phys. 6428

[23] Fleischer A, Wörner H J, Arissian L, Liu L R, Meckel M, Rippert A, Dörner R, Villeneuve D M, Corkum P B and Staudte A 2011 Probing angular correlations in sequential double ionization Phys. Rev. Lett. 107113003

[24] Hansen J L, Stapelfeldt H, Dimitrovski D, Abu-samha M, Martiny C P J and Madsen L B 2011 Time-resolved photoelectron angular distributions from strong-field ionization of rotating naphthalene molecules Phys. Rev. Lett. 106073001

[25] Remacle F and Levine R D 2006 An electronic time scale in chemistry Proc. Natl Acad. Sci. 1036793

[26] Lünnemann S, Kuleff A I and Cederbaum L S 2008 Charge migration following ionization in systems with chromophore-donor and amine-acceptor sites J. Chem. Phys. 129104305

[27] Popruzhenko S V, Paulus G G and Bauer D 2008 Coulomb-corrected quantum trajectories in strong-field ionization Phys. Rev. A 77053409 
[28] Arbó D G, Miraglia J E, Gravielle M S, Schiessl K, Persson E and Burgdörfer J 2008 Coulomb-Volkov approximation for near-threshold ionization by short laser pulses Phys. Rev. A 77013401

[29] Abu-samha M and Madsen L B 2011 Interrogation of orbital structure by elliptically polarized intense femtosecond laser pulses Phys. Rev. A 84023411

[30] Liu C and Hatsagortsyan K Z 2012 Coulomb focusing in above-threshold ionization in elliptically polarized midinfrared strong laser fields Phys. Rev. A 85023413

[31] Shvetsov-Shilovski N I, Dimitrovski D and Madsen L B 2012 Ionization in elliptically polarized pulses: multielectron polarization effects and asymmetry of photoelectron momentum distributions Phys. Rev. A 85023428

[32] Xie X et al 2012 Attosecond probe of valence-electronwave packets by subcycle sculpted laser fields Phys. Rev. Lett. 108193004

[33] Busuladžić M, Gazibegović-Busuladžić A and Milošević D B 2009 Strong-field approximation for ionization of a diatomic molecule by a strong laser field: part III. High-order above-threshold ionization by an elliptically polarized field Phys. Rev. A 80013420

[34] Odenweller M, Takemoto N, Vredenborg A, Cole K, Pahl K, Titze J, Schmidt L Ph H, Jahnke T, Dörner R and Becker A 2011 Strong field electron emission from fixed in space $\mathrm{H}_{2}{ }^{+}$ ions Phys. Rev. Lett. 107143004

[35] Yuan K J and Bandrauk A D 2011 Angle-dependent molecular above-threshold ionization with ultrashort intense linearly and circularly polarized laser pulses Phys. Rev. A 84013426

[36] Wu J, Meckel M, Voss S, Sann H, Kunitski M, Schmidt L Ph H, Czasch A, Kim H, Jahnke T and Dörner R 2012 Coulomb asymmetry in strong field multielectron ionization of diatomic molecules Phys. Rev. Lett. 108043002

[37] Yuan K J and Bandrauk A D 2012 Photoelectron angular distributions of $\mathrm{H}_{2}^{+}$and $\mathrm{HHe}^{2+}$ by intense circularly polarized extreme ultraviolet laser pulses J. Phys. B: At. Mol. Opt. Phys. 45105601

[38] Bucksbaum P H, Zavriyev A, Muller H G and Schumacher D W 1990 Softening of the $\mathrm{H}_{2}^{+}$molecular bond in intense laser fields Phys. Rev. Lett. 641883

[39] Giusti-Suzor A, He X, Atabek O and Mies F H 1990 Phys. Rev. Lett. 64515

[40] Zuo T and Bandrauk A D 1995 Phys. Rev. A 52 R2511

[41] Takemoto $\mathrm{N}$ and Becker A 2010 Multiple ionization bursts in laser-driven hydrogen molecular ion Phys. Rev. Lett. 105203004

[42] Posthumus J H 2004 Rep. Prog. Phys. 67623

[43] Saenz A 2000 Enhanced ionization of molecular hydrogen in very strong fields Phys. Rev. A 61051402

[44] Walsh T D G, Ilkov F A, Chin S L, Châteauneuf F, Nguyen-Dang T T, Chelkowski S, Bandrauk A D and Atabek O 1998 Laser-induced processes during the Coulomb explosion of $\mathrm{H}_{2}$ in a Ti-sapphire laser pulse Phys. Rev. A 583922

[45] Posthumus J H, Plumridge J, Taday P F, Sanderson J H, Langley A J, Codling K and Bryan W A 1999 Sub-pulselength time resolution of bond softening and Coulomb explosion using polarization control of laser-induced alignment J. Phys. B: At. Mol. Opt. Phys. $32 \mathrm{~L} 93$

[46] Alnaser A S et al 2004 Routes to control of $\mathrm{H}_{2}$ Coulomb explosion in few-cycle laser pulses Phys. Rev. Lett. 93183202
[47] Rudenko A, Feuerstein B, Zrost K, de Jesus V L B, Ergler T, Dimopoulou C, Schröter C D, Moshammer R and Ullrich J 2005 Fragmentation dynamics of molecular hydrogen in strong ultrashort laser pulses J. Phys. B: At. Mol. Opt. Phys. 38487

[48] Ergler Th, Rudenko A, Feuerstein B, Zrost K, Schröter C D, Moshammer R and Ullrich J 2005 Time-resolved imaging and manipulation of $\mathrm{H}_{2}$ fragmentation in intense laser fields Phys. Rev. Lett. 95093001

[49] Niikura H, Villeneuve D M and Corkum P B 2006 Controlling vibrational wave packets with intense, few-cycle laser pulses Phys. Rev. A 73021402

[50] Staudte A et al 2007 Attosecond strobing of two-surface population dynamics in dissociating $\mathrm{H}_{2}^{+}$Phys. Rev. Lett. 98073003

[51] Bocharova I A, Alnaser A S, Thumm U, Niederhausen T, Ray D, Cocke C L and Litvinyuk I V 2011 Time-resolved Coulomb-explosion imaging of nuclear wave-packet dynamics induced in diatomic molecules by intense few-cycle laser pulses Phys. Rev. A 83013417

[52] Roither S et al 2011 High energy proton ejection from hydrocarbon molecules driven by highly efficient field ionization Phys. Rev. Lett. 106163001

[53] Ullrich J, Moshammer R, Dorn A, Dörner R, Schmidt L Ph H and Schmidt-Böcking H 2003 Recoil-ion and electron momentum spectroscopy: reaction-microscopes Rep. Prog. Phys. 661463

[54] Chelkowski S, Bandrauk A D, Staudte A and Corkum P B 2007 Dynamic nuclear interference structures in the Coulomb explosion spectra of a hydrogen molecule in intense laser fields: re-examination of molecular enhanced ionization Phys. Rev. A 76013405

[55] Gibson G N, Li M, Guo C and Neira J 1997 Strong-field dissociation and ionization of $\mathrm{H}_{2}^{+}$using ultrashort laser pulses Phys. Rev. Lett. 792022

[56] Ben-Itzhak I et al 2008 Elusive enhanced ionization structure for $\mathrm{H}_{2}^{+}$in intense ultrashort laser pulses Phys. Rev. A 78063419

[57] Alnaser A S, Tong X M, Osipov T, Voss S, Maharjan C M, Shan B, Chang Z and Cocke C L 2004 Laser-peak-intensity calibration using recoil-ion momentum imaging Phys. Rev. A 70023413

[58] Smeenk C, Salvail J Z, Arissian L, Corkum P B, Hebeisen C T and Staudte A 2011 Precise in situ measurement of laser pulse intensity using strong field ionization Opt. Express 199336

[59] Pfeiffer A N, Cirelli C, Landsman A, Smolarski M, Dimitrovski D, Madsen L B and Keller U 2011 Probing the longitudinal momentum spread of the electron wave packet at the tunnel exit arXiv:1111.6033v2[physics.atom-ph]

[60] Reid K L, Leahy D J and Zare R N 1992 Complete description of molecular photoionization from circular dichroism of rotationally resolved photoelectron angular distributions Phys. Rev. Lett. 683527

[61] Jahnke T et al 2002 Circular dichroism in k-shell ionization from fixed-in-space $\mathrm{CO}$ and $\mathrm{N}_{2}$ molecules Phys. Rev. Lett. 88073002

[62] Dowek D, Pérez-Torres J F, Picard Y J, Billaud P, Elkharrat C, Houver J C, Sanz-Vicario J L and Martin F 2010 Circular dichroism in photoionization of $\mathrm{H}_{2}$ Phys. Rev. Lett. 104233003

[63] Corkum P B, Burnett N H and Brunel F 1989 Above-threshold ionization in the long-wavelength limit Phys. Rev. Lett. 621259

[64] Smeenk C T L, Arissian L, Zhou B, Mysyrowicz A, Villeneuve D M, Staudte A and Corkum P B 2011 Partitioning of the linear photon momentum in multiphoton ionization Phys. Rev. Lett. 106193002 